\title{
Unravelling the disease mechanism for TSPYL1 deficiency
}

Gunnar Buyse ${ }^{1}$, Michela Di Michele ${ }^{2}$, Anouck Wijgaerts ${ }^{3}$, Sophie Louwette ${ }^{3}$, Christine Wittevrongel ${ }^{3}$, Chantal Thys ${ }^{3}$, Kate Downes ${ }^{4,5}$, Berten Ceulemans ${ }^{6}$, Hilde Van Esch $^{7,8}$, Chris Van Geet $^{1}$, Kathleen Freson ${ }^{3 *}$

${ }^{1}$ Department of pediatric neurology, University Hospitals Leuven, Leuven, Belgium

${ }^{2}$ Institut des Biomolécules Max Mousseron (IBMM), UMR 5247, Université de Montpellier, Ecole Nationale Supérieure de Chimie de Montpellier, Montpellier, France

${ }^{3}$ Department of Cardiovascular Sciences, Center for Molecular and Vascular Biology, University of Leuven, Leuven, Belgium

${ }^{4}$ East Genomic Laboratory Hub, Cambridge University Hospitals NHS Foundation Trust, Cambridge, CB2 0QQ, UK

${ }^{5}$ Department of Haematology, University of Cambridge, Cambridge Biomedical Campus, Cambridge, CB2 0PT, UK

${ }^{6}$ Department of pediatric neurology, University hospital, University of Antwerp, Antwerp, Belgium

${ }^{7}$ Center for Human Genetics, University Hospitals Leuven, Leuven, Belgium

${ }^{8}$ Laboratory for the Genetics of Cognition, Department of Human Genetics, KU Leuven, Leuven, Belgium.

(C) The Author(s) 2020. Published by Oxford University Press. All rights reserved. For Permissions, please email: journals.permissions@oup.com 
Address for correspondence: *Kathleen Freson, Center for Molecular and Vascular Biology, University of Leuven, Campus Gasthuisberg, O\&N1, Herestraat 49, Box 911, 3000 Leuven, Belgium; e-mail: kathleen.freson@kuleuven.be; Tel: 32-16322707, Fax: 32-16345990

\section{Abstract}

We describe a lethal combined nervous and reproductive systems disease in three affected siblings of a consanguineous family. The phenotype was characterized by visceroautonomic dysfunction (neonatal bradycardia/apnea, feeding problems, hyperactive startle reflex), severe postnatal progressive neurological abnormalities (including abnormal neonatal cry, hypotonia, epilepsy, polyneuropathy, cerebral gray matter atrophy), visual impairment, testicular dysgenesis in males, and sudden death at infant age by brainstem-mediated cardiorespiratory arrest. Whole exome sequencing revealed a novel homozygous frameshift variant p.Val242GlufsTer52 in the TSPY-like 1 gene TSPYL1. The truncated TSPYL1 protein that lacks the nucleosome assembly protein (NAP) domain was retained in the Golgi of fibroblasts from the three patients while control fibroblasts express full length TSPYL1 in the nucleus. Proteomic analysis of nuclear extracts from fibroblasts identified 24 up- and 20 down-regulated proteins in the patients compared to five controls with "regulation of cell cycle" as the highest scored biological pathway 
affected. TSPYL1 deficient cells had prolonged S and G2 phases with reduced cellular proliferation rates. Tspyl1 depletion in zebrafish mimicked the patients' phenotype with early lethality, defects in neurogenesis and cardiac dilation. In conclusion, this study reports the third pedigree with recessive TSPYL1 variants, confirming that TSPYL1 deficiency leads to a combined nervous and reproductive systems disease, and provides for the first time insights into the disease mechanism.

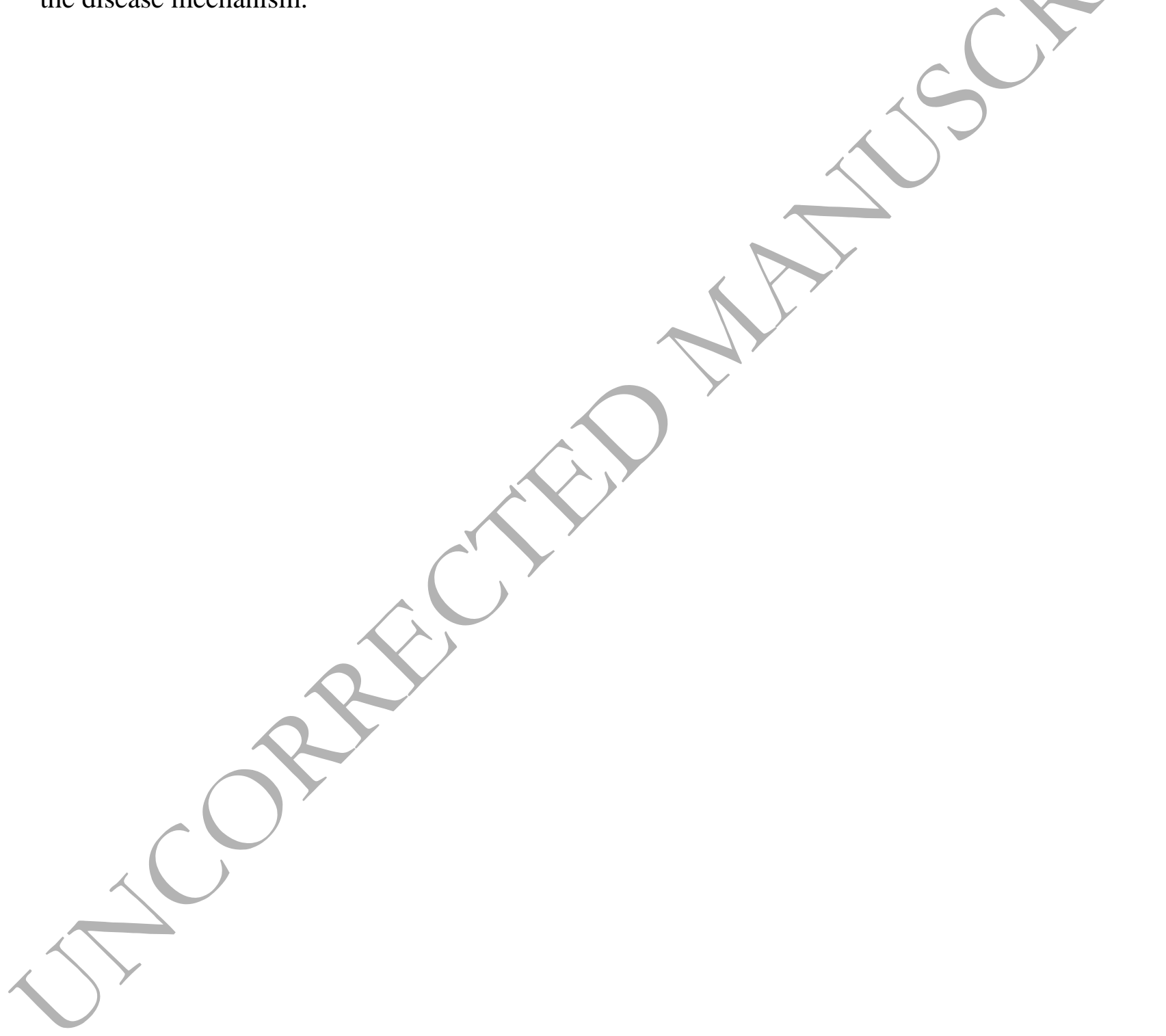




\section{Introduction}

TSPY/TSPYL/SET/NAP1 (TTSN) superfamily proteins contain a nucleosome assembly protein (NAP) domain $(1,2)$. Members of this family execute divers functions, including cell cycle regulation, nucleosome assembly via histone binding, DNA replication, transcription and translation by direct protein interaction via their NAP domain. The best-characterized protein of all members is yeast NAP1, a 48-kDa polypeptide that binds histones and mediates nucleosome assembly (3). Human NAP1, encoded by NAP1L1, shares $54 \%$ amino acid similarity to yeast NAP1 and was found to participate in DNA replication and cell proliferation (4). Fluorescently tagged NAP1 is located in the nucleus in the $\mathrm{S}$ phase while it was found in the cytoplasm during the $G_{2}$ phase of the cell cycle, suggesting that NAP1 may be involved in shuttling histones between the cytoplasm and the nucleus (5).

Though many studies have illustrated the role of TTSN proteins in diverse cellular functions, our knowledge about their contribution to human pathology is very limited. Exome sequencing has recently detected autosomal dominant variants in SET, the gene for the SET nuclear protooncogene, as cause for non-syndromic intellectual disability in five unrelated patients (6). Two patients carried a missense variant in the NAP domain of SET while the others had frameshift variants. Functional studies using patient-derived cells were not performed but literature-based evidence for a role of SET in neurogenesis was provided $(7,8)$. Knockout mice for Set die during embryonic development between days 11.5 and 12.5 and show multiple developmental defects such as cardiac edema and an open neural tube (9). In 2004, a homozygous frameshift variant in the TSPY-like 1 gene TSPYL1 (MIM604714) was detected using linkage analysis and candidate gene Sanger sequencing in a large Amish pedigree where 21 children suffered from an autosomal recessive lethal disease characterized by sudden infant death 
(from cardiac and respiratory arrest) with dysgenesis of the testes (SIDDT) in males (MIM608800) (10). This variant was predicted to result in a shorter protein that lacks the NAP domain and transfection studies in HELA cells showed how this truncated protein remained in the cytoplasm while wild type TSPYL1 was detected in the nucleus (10). No additional functional studies were performed and it was concluded that further elucidation of TSPYL1 function could shed light on the molecular control of embryogenesis of the nervous and reproductive systems via a complete novel pathway. A follow-up genetic study suggested a link between heterozygous missense variants in TSPYL1 and anomalies of testicular development and function (11), but such association could not be confirmed by another study (12). Other studies did not find an association between TSPYL1 variants and patient cohorts with isolated (i.e. otherwise normal children) sudden infant death $(13,14)$. Only very recently, a homozygous TSPYL1 frameshift variant was found in a girl diagnosed with SIDDT in a second non-Amish pedigree (15). This patient also suffered from mild T-cell lymphopenia and developed intractable epilepsy. Functional studies to unravel a role of TSPYL1 in the pathogenesis were again not performed in this case report.

With this study, we support the causal association between recessive loss of function variants in TSPYL1 and a progressive combined nervous and reproductive systems disease including sudden infant death plus testicular dysgenesis in males. The functional impact of TSPYL1 depletion was studied by using two combined models, fibroblasts derived from three patients and zebrafish embryos. The homozygous frameshift variant resulted in a shorter TSPYL1 protein that retained in the Golgi from patients' fibroblasts and their proteome analysis pointed to a defect in cell cycle regulation. Tspyl1 depletion in zebrafish embryos mimicked phenotypes present in the patients.

\section{Results}




\section{Clinical phenotype of affected patients}

The consanguineous Turkish pedigree includes three affected siblings (P1, P2 and P4) who died of sudden cardiorespiratory arrest at the ages of 5, 9 and 5 months, respectively, and one healthy sibling (P3) (figure 1A). The healthy parents are second degree relatives. Patient 1 (P1; born in 2001) was a boy that presented at neonatal age with hypotonia, hypoventilation and obstructive and central apneas, swallow and feeding problems, hypogonadism, microgenitalia, and a progressive neurological disorder with regression, epilepsy and delayed visual development. Magnetic resonance imaging at 6 months revealed cerebral (gray matter) degeneration with evidence for poliodystrophy. He died at 9 months (sudden death by cardiorespiratory arrest). The phenotype of patient 2 (P2; born in 2004) was similar to that of his older sibling P1. He presented at neonatal age with bradycardia, hypoventilation and apneas (obstructive and central), abnormal cry (weak high pitched) and neurological behavior with excessive startle reflex and hypotonia, epilepsy (multifocal, seizure free under anti-epileptic treatment), delayed visual development, axonal polyneuropathy, severe feeding problems (tube feeding) and failure to thrive, and hypogonadism (vanishing testes) and microgenitalia. No structural brain (MRI at 3 weeks) or heart defects could be noticed. He died at 6 months (sudden death by cardiorespiratory arrest). The third affected sibling (P4; female, born in 2012) had an identical phenotype as her affected brothers, although slightly less severe (some motor development present). Prior to birth at postmenstrual age 38 weeks fetal bradycardias were observed. Her external genitalia were normal.

She died at the age of 5 months (sudden death).

Whole exome sequencing identifies a homozygous TSPYL1 frameshift variant 
Whole exome sequencing combined with homozygosity mapping for P2 revealed seven possible candidate variants (Supplementary Table 1). After validation of these homozygous variants in the affected and non-affected siblings, the variant in TSPYL1 remained as the only possible candidate for the disorder. Patients P1, P2 and P4 were homozygous for the TSPYL1 frameshift variant c.725_726 delTG while parents and the non-affected sibling P3 were heterozygous for this variant (Figure 1A). This variant (p.Val242GluTer52) results in a shorter protein of 292 amino acids compared to the normal 437 amino acid wild type TSPYL1 protein (Figure 1B). As TSPYL1 is a single exon gene, the frameshift resulted in the generation of a truncated protein rather than leading to non-sense mediated decay. The mutant protein lacks the complete NAP domain and is thus predicted to localize in the cytoplasm instead of the cellular nucleus (10). The minor allele frequency of this frameshift variant in the gnomAD population database was $0.0021 \%$ but no homozygous carrier could be detected. Moreover, no homozygous Loss Of Function (LOF) variants in TSPYL1 are repørted in gnomAD.

\section{TSPYL1 retention in Golgi of patients' fibroblasts}

Protein expression studies using immunoblot analysis of subcellular protein extracts showed expression of TSPYL1 in the nuclear extract of fibroblasts from three unrelated healthy subjects (Figure 1C and Supplementary Figure 1). In contrast, fibroblasts of the three patients showed expression of a shorter TSPYL1 protein in the cytosol extracts. Further detailed subcellular expression studies were performed by immunostaining. While wild type TSPYL1 is expressed in the nucleus and weakly in the Golgi of normal fibroblasts, the shorter mutant TSPYL1 is mainly retained in the Golgi of the patients' fibroblasts (Figure 1D, Supplementary Figure 2). 


\section{Proteomic analysis of nuclear extracts from control and TSPYL1 deficient fibroblasts}

2D-DIGE was used to analyze nuclear extracts from fibroblasts of five unrelated controls and the three patients (Supplementary figure 3). Equal amounts of proteins from control and patient nuclear extracts were labelled with $\mathrm{Cy} 3$ or $\mathrm{Cy} 5$ dyes, respectively. The quantitative comparison for a protein of the two samples co-resolved on the same gel was between the Cy3 or Cy 5 signals, while the $\mathrm{Cy} 2$ was used as internal standard signal for this protein. Representative DIGE gels for proteins detected in nuclear extracts from a control and patient are shown in Supplementary figure 3 . The Cy2, Cy3, and Cy5 channels of each gel were individually imaged and the comparisons of protein expression in 2D images were carried out using Decyder software. One-way ANOVA was performed on the normalized abundances of matched spots to compare similarity between the patients and controls according to expression patterns. Unsupervised Principle Component Analysis of the protein expression levels within each sample clearly separates the proteome of patients from controls (Supplementary figure 3).

Comparison between patient and control gels revealed that 48 protein spots varied in a statistically significant way $(p<0.05)$ with a patient/control fold change of at least 1.2 (Figure 2A). MALDI TOF-TOF MS analysis could identify 44 out of the 48 protein spots and the results of protein identifications are listed in Table 1 . Several proteins were identified at multiple positions on the gel, suggestive for post-translational modifications and different isoforms. The final number of uniquely identified proteins modified in patients was 35 of which 15 were previously identified in cell nucleus. STRING analysis of these 15 nuclear proteins showed that most differentially expressed proteins regulate the cell cycle, are involved in cardiomyopathy and are located in the nuclear lumen (Supplementary table 2). Ingenuity Pathway Analysis software 
was used to determine the most significant interaction networks and the "cell cycle" pathway resulted as most significant (Figure $2 \mathrm{~B}$ ).

Cell cycle and proliferation defect in TSPYL1 deficient fibroblasts

The patients' fibroblasts express a mutant protein that resides in the Golgi and therefore it is predicted that this protein cannot fulfill its normal function as regulator of cell cycling and nucleosome assembly. Cell cycle studies were performed by flow cytometry and significantly reduced G1 and increased S and G2 phases were detected in the patients' fibroblasts compared to controls (Figure 2C and D). Since a G2/M arrest can result in decreased cell proliferation, this was further studied. The patients' fibroblasts indeed showed a/significant decreased cell proliferation rate compared to controls (Figure 2E).

\section{Phenotype description of TSPYL1 depleted zebrafish embryos}

ENSDART00000007482 is the orthologue gene for human TSPYL1 in zebrafish. Tspyll depletion in zebrafish was obtained using a splice morpholino (tspyl1-MO) that resulted in intron 4/5 retention (Supplementary figure 4). Tspyl1 depleted embryos present with a deformed axis between 2 and $3 \mathrm{dpf}$ (Figure $3 \mathrm{~A}$ and $\mathrm{B}$ ). These embryos were unable to independently remove their yolk sac and 50\% died between 3 and 5 dpf (Figure 3C). In situ hybridization of wholemount 1dpf embryos with probes for $\lim 3$ and $\operatorname{slc} 35 \mathrm{~d} 3$ showed a normal pituitary gland development (Supplementary figure 5) while staining for pax2a showed a complete absence of the optic stalk in tspyll depleted embryos (Supplementary figure 6). Tspyll depleted embryos

have smaller heads and eyes. The formation of the optic stalk is part of the retinal neurogenesis in vertebrates and the expression of the proneural gene atonal-homologue 5 (ath5) is closely 
associated with the activation of retinal neurogenesis in all vertebrates $(19,22)$. Transgenic $\mathrm{Tg}$ (Ath5:eGFP) embryos were used to characterize the development of the optic tectum and tspyl1 depleted embryos at $5 \mathrm{dpf}$ showed a significantly smaller optic tectum compared to control embryos, when corrected for the head size (Figures 3D and E). Transgenic Tg(cmcl2:eGFP) embryos carrying eGFP driven by heart-specific cmlc2 promoter were used to study heart development (18). At 3dpf, tspyll depleted embryos present with heart edema (Figures 3F and G) and dilated hearts (Figure 3H) with inverted looping and bradycardia (Figures $3 \mathrm{H}$ and $\mathrm{I}$ ).

\section{Discussion}

The first causal association between the TSPYL1 gene and human disease was reported in 2004, with the identification of a homozygous frameshift variant (c.457-458insG) in patients from a single Amish pedigree with a phenotype that included sudden infant death and dysgenesis of the testes syndrome in neurologically abnormal infants (MIM608800) (10). Our study now reports a recessive pathogenic TSPYL1 variant in three siblings with a phenotype comparable to the Amish pedigree, confirming that TSPYL1 deficiency leads to a combined nervous and reproductive systems disease. Our findings also provide for the first time insights into the disease mechanism. Patient fibroblast studies showed a Golgi (instead of the normal nuclear) localization of the mutated truncated TSPYL1 protein, with consequent cell cycle dysregulation and cell proliferation defects.

The clinical phenotype in the Amish pedigree involved visceroautonomic nerve dysfunction early in life, with death due to abrupt cardiorespiratory arrest before 12 months of age. It included neonatal bradycardia, hypothermia, feeding problems with severe gastroesophageal reflux, 
respiratory problems with laryngo/bronchospasms and apneas, abnormal cardiorespiratory pattern during sleep, hyperactive startle reflex, plus fetal testicular dysgenesis and ambiguous genitalia in males. The phenotype of the three affected siblings reported in this study was characterized by visceroautonomic dysfunction, bradycardias and hypoventilation/apneas, severe postnatal progressive neurological abnormalities (including abnormal neonatal cry and neurological behavior, hypotonia, epilepsy, cerebral gray matter atrophy), visual impairment, testicular dysgenesis in males, and sudden death at infant age by brainstem-mediated cardiorespiratory arrest. This indicates that recessive loss of function variants in TSPYLI are responsible for this severe and lethal progressive combined nervous and reproductive systems disease, where TSPYL1 function seems crucial for the development or function of the brainstem as this regulates basic body functions including heart rate and breathing.

Decreased survival with cardiac edema and dysfunction as well as defects in neurogenesis were also present in tspyll depleted zebrafish. Interestingly, proteomic analysis of nuclear extracts from skin fibroblasts of the affected patients also identified 'hypertrophic cardiomyopathy' and 'dilated cardiomyopathy' among the highest scored dysregulated pathways by performing enrichment analysis It was previously shown that downregulation of the nucleosome assembly protein 1-like 1(Nap111) significantly enhances mesodermal induction and subsequent cardiogenesis of murine-induced pluripotent stem cells via inhibition of Notch signaling (23). More recently, it was found that nap 111 expression in zebrafish embryos between 1 and 3 dpf maintains throughout the tectum, olfactory vesicle, lens, optic cups, heart, branchial arches, pectoral fins, axial vasculature, pronephros, and lateral line neuromasts (24). Expression studies of tspyll in zebrafish embryos at $3 \mathrm{dpf}$ using WISH showed expression in the brain, eye, ear, fin 
and heart (data not shown). Further studies are required to define the exact role of TSPYL1 in cardiac function, but neural control could be involved. The optic tectum in zebrafish embryos has emerged as a valuable system to examine neural circuits. The optic tectum is a multilaminated structure in which tectal cell dendrites receive synapses from sensory afferents and interneurons (25). Transgenic Tg (Ath5:eGFP) zebrafish embryos have been developed to study tectal circuits as they label retinal ganglion cell axons that connect the eye to the brain (22). Tspyll depleted zebrafish showed significantly reduced retinal ganglion cell axons numbers in the optic tectum.

Our study also focused on the identification of the underlying pathological pathway for this disease and could provide evidence for an important role of TSPYL1 in cell cycle regulation and proliferation. Fibroblasts from patients had a reduced cell cycle G1 phase while the S and G2 phases were prolonged. The biological role of TSPYL1/was never investigated before, but a previous study showed that TSPYL2 contro1 $\widehat{\mathrm{s}}$ the $\mathrm{G} 1$ checkpoint after DNA damage via gamma irradiation of mouse embryonic fibroblasts using Tspyl2 knockout mice (26). The SET protein was also previously shown to regulate G2/M transition by inhibition of cyclin B-CDK1 activity (27). Most TTSN proteins interact with nucleosomes via their NAP domain but have also been shown to interact with cyclin B (28-30). Cyclin B normally increases in the late S phase, peaks in the G2 phase and mitorsis, and decreases during the early G1 phase (31). Interestingly, the proteomic data in the current study showed significantly elevated cyclin B3 (CCNB3) expression in nuclean extracts from the patients' fibroblasts that could be compatible with prolonged S/G2 phases. In addition, differential regulation of the cell cycle was detected as the most significant pathway in the proteomic analysis of the nuclear extracts of the patients' fibroblasts. TSPYL1 (or referred to as Q9H0U9) was previously identified as one of the 213 different nucleolar proteins 
using proteomics, but was assigned in that study to the class of proteins with an unknown function (32). Additional studies are required to determine the exact role of TSPYL1 in cell cycle regulation. Defects in cell cycle regulation have been implicated in the pathogenesis of diverse multifactorial diseases, such as cancer, myocardial infarction, stroke, atherosclerosis, infection, inflammation and neurodegenerative disorders (including Alzheimer's disease) (33), but their contribution to rare Mendelian syndromes remains very limited. A recent study introduced the term 'cell cycle-opathy' for Mendelian disorders characterized by defects in DNA replication or cell cycle in patients that typically present with primordial dwarfism and microcephaly together with particular skeletal abnormalities (34).

In conclusion, with the description of three patients from a second non-Amish pedigree, this study confirms the role of TSPYL1 deficiency as cause of a progressive combined nervous and reproductive systems disease. Our data suggests that TSPYL1 deficiency probably results in a human disease via alterations in the regulation of the cell cycle. 


\section{Material and methods}

\section{Patient study}

Informed consent was obtained from the legal representative (parents) of the children. The Ethics Committee of UZ Leuven approved the study (ML3580).

Exome and Sanger sequencing

Whole exome sequencing and variant filtering was performed as described (16). Sanger sequencing of PCR amplified fragments was used to validate the homozygous yariants in TSPYL1, ENPP3, SYNC, MRPS15, CACNA1C, PAN3 and HR (primers available upon request). The NCBI reference sequence for TSPYL1 is NM_003309.4.

\section{Antibodies}

The following antibodies were used: mouse polyclonal against human TSPYL1 (MaxPab H00007259-B01P; Abnova used at 1:1000 for immunoblot and 1:50 for immunostaining), rabbit polyclonal against human HDAC1 (Clone $\hat{H}_{-}$-51; Santa Cruz used at 1:1000 for immunoblot), rabbit monoclonal human beta-actin (Clone 13E5, Cell Signaling Technology used at 1:1000 for immunoblot) and rabbit polyclonal golgi marker Mannosidase II (Abcam used at 1:200 for immunostaining).

\section{Human skin fibroblasts}

Skin fibroblasts were obtained via punch biopsy from the volar side of the upper arm from patients $\mathrm{P} 1, \mathrm{P} 2$ and $\mathrm{P} 4$ and normal healthy subjects $\mathrm{C} 1, \mathrm{C} 2, \mathrm{C} 3, \mathrm{C} 4$ and $\mathrm{C} 5$. Fibroblasts were cultured in DMEM/H12 (Life Technologies) supplemented with $10 \%$ fetal bovine serum and antibiotics (Life Technologies). Only fibroblasts of low passage number (between 6 and 12) were used for experiments.

Flow Cytometry to analyze cell cycling 
Fibroblasts were plated (200.000 cells/6-well) and trypsinized after 24 hours, washed with PBS and fixed with ice-cold $70 \%$ ethanol for $30 \mathrm{~min}$ at $4{ }^{\circ} \mathrm{C}$. Cells were washed with PBS and resuspended in $400 \mu \mathrm{L}$ staining solution $(20 \mathrm{u} \mu \mathrm{g} / \mathrm{ml}$ RNase inhibitor, $50 \mu \mathrm{g} / \mathrm{ml}$ propidium iodide in PBS) for 30 min at room temperature before flow cytometric analysis. We used Cell Diva software for two-color immunofluorescence acquisition on a FACS Canto II flow cytometer (BD Biosciences, San Jose, CA, USA) and for data analysis to determine the percentage of cells in G1, S and G2 phases. All experiments were performed in triplicate.

Immunostaining analysis

Adherent fibroblasts were washed and fixed with $4 \%$ paraformaldehyde in cytoskeleton buffer (0.1M PIPES, 2M glycerol, 1mM EDTA, 1mM MgCl2, $\mathrm{pH}$ 6.9), and permeabilized for 15 minutes with $0.2 \%$ triton $\mathrm{X}-100$ (Roche) at room temperature. After blocking with $1 \%$ bovine serum albumin (BSA) (Albumax, Life Technologies) for 30 minutes at room temperature, cells were incubated with a diluted primary anti-antibody overnight at $4^{\circ} \mathrm{C}$. After 5 washing steps with PBS, cells were incubated with a specific secondary antibody diluted 1:200 (Alexa Fluor 488 or 568 or 647 conjugated; Life technologies) for $45 \mathrm{~min}$ at $37^{\circ} \mathrm{C}$. Images were made with the Zeiss Elyra 5.1 (VIB BioImaging Core facility, KULeuven) for TSPYL1/mannosidase staining and with a Zeiss Axiovert microscope and captured with Zeiss AxioVision for TSPYL1/mannosidase/DAPI staining.

Immunoblot analysis

Cytosolic and nuclear fractions from fibroblast lysates were obtained by using the subcellular protein fractionation kit (Thermo Scientific) according to the instructions of the manufacturer.

Protein fractions were resolved by SDS/PAGE and transferred onto a nitrocellulose membrane. Membranes were blocked in 5\% milk powder in Tris-buffered saline-Tween 20 (TBST; $10 \mathrm{~mm}$ 
Tris- $\mathrm{HCl} \mathrm{pH} 8.0,150 \mathrm{~mm}$ sodium chloride, $0,1 \%$ Tween 20 ) for $1 \mathrm{~h}$ and then incubated overnight at $4{ }^{\circ} \mathrm{C}$ with the primary antibodies. Membranes were next incubated with HRP-conjugated secondary antibody and developed by using the ECL detection reagent. Protein bands were quantified using the Bio-Rad Molecular Imager Chemi Doc ${ }^{\mathrm{TM}}$ XRS and Image Lab software version 4.0. All experiments were performed in triplicate.

Proteomic analysis of nuclear extracts by 2D-Differential In-Gel Electrophoresis (DIGE) and MS/MS analysis

Nuclear fractions from patients and controls were solubilized in sample buffer $(7 \mathrm{M}$ urea, $2 \mathrm{M}$ thiourea, 4\% CHAPS, $30 \mathrm{mM}$ Tris, $\mathrm{pH} 8.5$ ) containing a mixture of protease inhibitors (Complete protease inhibitor, Roche Diagnostics). The protein concentration was determined by the Bio-Rad Dc protein assay (BioRad, Hercules, CA). Sample proteins $(50 \mu \mathrm{g})$ were minimally labeled with 400 pmol of Cy3 or Cy5 (GE Healthcare), whereas the pooled internal standard was labeled with Cy2. The internal standard was made up of equivalent amounts of all the 8 samples. The labelling reaction, the first and second dimension and the labelled protein spot visualization were carried out as previously described (17). Briefly, gel analysis was performed using DeCyder 2-D Differential Analysis Software v6.5 (Amersham GE Healthcare). The EDA (Extended Data Analysis) module carried out intra- and inter-gel statistical analyses, performing expression pattern clustering based on PCA and ANOVA. The protein spots having a p-value $<0.05$ for the T-test, by applying the false discovery rate (FDR) correction method and differentially expressed between patient and controls with a fold-change of at least 1.2 were identified by mass spectrometry. To this aim, after image acquisition, gels were fixed in 50\% methanol, $7 \%$ acetic acid for $1 \mathrm{~h}$ and then visualized with silver staining to pick spots of interest. 
Mass spectrometry analysis was performed as described (17). In brief, gel pieces were cut out of a preparative gel run with $300 \mu \mathrm{g}$ of proteins and destained. Resulting trypsin digested peptides were concentrated and desalted by using Millipore C18 ZipTips (Millipore, Bedford, MA, USA). The samples were mixed in a 1:1 (v/v) ratio with alpha-cyano-4-hydroxycinnamic acid matrix and spotted onto the MALDI target plate. MS/MS analyses were performed on a 4800 MALDITOF/TOF instrument (Applied Biosystems, Foster City, CA). Data interpretation was carried out using the GPS Explorer software v3.5 and database searching was performed using the Mascot program v2.0.00. MS/MS searches were conducted with the following settings: NCBI and MSDB (taxonomy set on humans) as database, MS/MS tolerance for precursor and fragment ions between 0.2 and 1Da depending on the sample, carbamidomethylation of cysteine as fixed modification, methionine oxidation as variable modification, one missed cleavage allowed in case of incomplete trypsin hydrolysis. Using these parameters the probability-based MOWSE (Molecular Weight Search) scores greater than the given cutoff value for MS/MS fragmentation data were taken as significant $(\mathrm{p}<0.05)$.

Fibroblast proliferation assay

Fibroblasts were plated and analyzed for proliferation efficiency by using the Quick Cell proliferation assay kit (Abcam) according to the protocol of the manufacturer. All experiments were performed in triplicate.

Zebrafish injections

The ZFIN reference sequence for zebrafish Tspyll is ZDB-GENE-030131-9158. Zebrafish embryos were injected at the 1-cell stage with splice morpholino (tspyl1-MO) CATGAGAGATGTACCTGTGCTATGT (located in exon 4 and intron 4/5) at $400 \mu \mathrm{M}$. Offtarget effects were assessed by including a standard control MO (against beta-globin 
CCTCTTACCTCAGTTACAATTTATA) at $400 \mu \mathrm{M}$. MO were designed by Gene-Tools LLC (Philomath,OR). Embryos were life-screened from 1 to 5 days post fertilization (dpf) using a Zeiss Lumar V12 and images were captured with a Zeiss Axiocam MRc camera using AxioVision software (Carl Zeiss, Jena, Germany). All animal protocols were approved by the Ethical Committee of the KULeuven. AB wild type and $\operatorname{Tg}(\mathrm{Cmcl2}: e G F P$ ) (gift from Dr HJ. Tsai, Institute of Biomedical Science, Mackay Medical College, Taiwan, 17) and $\operatorname{Tg}($ Ath5:eGFP) (gift from Dr S Wilson, Developmental Biology Research Centre, Randall Institute, King's College, UK, 19) transgenic zebrafish lines were used.

Whole-Mount In Situ Hybridization (WISH) of zebrafish embryos

Total RNA was extracted from zebrafish embryos at various stages of development to amplify a RT-PCR fragment of the zebrafish orthologues for $\lim 3$ (20), slc35d3 and pax $2 \mathrm{a}$ (primers available upon request). These fragments were cloned into pGEM-T-easy (Promega) for making antisense probes by linearization of the plasmid and subjected to in vitro transcription using the mMessage mMachine High Yield Capped RNA kit (Ambion). Developmental staging of injected zebrafish was determined by somite number and embryos were fixed in $4 \%$ paraformaldehyde. Assays for RNA expression using WISH were performed as described (21). Embryos were visualized with a Zeiss Lumar V12 or a Zeiss Axiovert microscope and images were captured with a Zeiss Axiocam MR camera using AxioVision software.

\section{Quantification of zebrafish optic tectum}

The size of the optic tectum was measured using the $\operatorname{Tg}$ (ath5:eGFP) transgenic line as described elsewhere (22). 5dpf images of dorsal views were captured with a Zeiss Lumar 100M microscope and the diameter of the optic tectum as well as the total diameter of the head were measured using AxioVision software. The ratio optic tectum/head size was assessed and p values were 
calculated based on Mann Whitney test. Additional confocal images were captured on the Olympus FV1000.

\section{Acknowledgements}

This study makes use of data generated by the NIHR BioResource-Rare Disease Consortium.

This work was supported by KU Leuven BOF grant C14/19/096 and research grants from Sobi,

CSL Behring and Bayer. GB is senior clinical investigator of the Research Foundation Flanders (FWO Vlaanderen, Belgium).

\section{Conflict of Interest Statement}

The authors declare no conflict of interest.

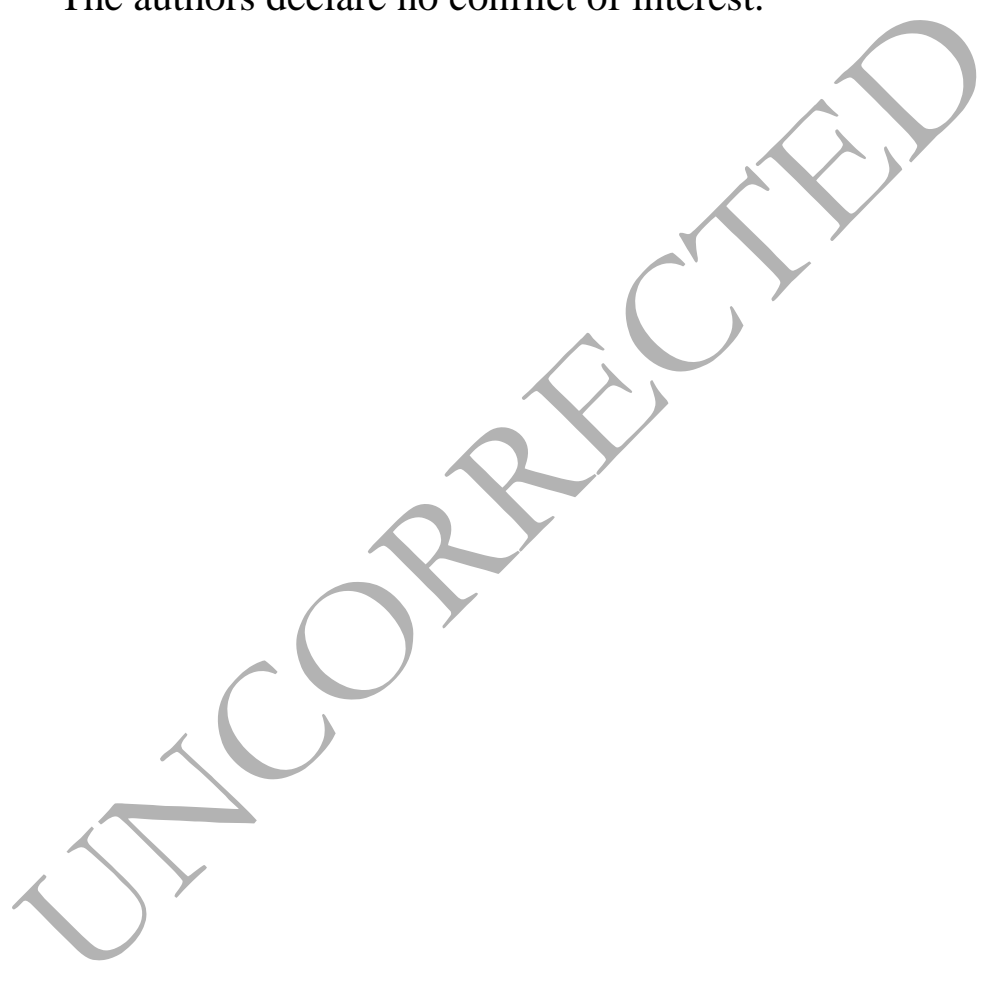




\section{References}

1. Vogel, T., Dittrich, O., Mehraein, Y., Dechend, F., Schnieders, F. and Schmidtke, J. (1998) Murine and human TSPYL genes: novel members of the TSPY-SET-NAP1L1 family. Cytogenet. Cell. Genet., 81, 265-270.

2. Park, Y.J. and Luger, K. (2006) Structure and function of nucleosome assembly proteins. Biochem. Cell. Biol., 84, 549-558.

3. Ishimi, Y. and Kikuchi, A.J (1991) Identification and molecular cloning of yeast homolog of nucleosome assembly protein I which facilitates nucleosome assembly in vitro. Biol Chem., 266, 7025-7029.

4. Simon, H.U., Mills, G.B., Kozlowski, M., Hogg, D., Branch, D., Ishimi, Y. and Siminovitch, K.A. (1994) Molecular characterization of hNRP, a cDNA encoding a human nucleosome-assembly-protein-L-related gene product involved in the induction of cell proliferation. Biochem. J., 297, 389-397.

5. Marheineke, K. and Krude T.J. (1998) Nucleosome assembly activity and intracellular localization of human CAF-1 changes during the cell division cycle. Biol. Chem., 273, $15279-15286$.

6. Stevens, S.J.C., van der Schoot, V., Leduc, M.S., Rinne, T., Lalani, S.R., Weiss, M.M., van Hagen, J.M., Lachmeijer, A.M.A.; CAUSES Study, Stockler-Ipsiroglu, S.G., et al. (2018) De novo mutations in the SET nuclear proto-oncogene, encoding a component of the inhibitor of histone acetyltransferases (INHAT) complex in patients with nonsyndromic intellectual disability. Hum. Mutat., 39, 1014-1023. 
7. Qu, D., Zhang, Y., Ma, J., Guo, K., Li, R., Yin, Y., Cao, X. and Park, D.S. (2007) The nuclear localization of SET mediated by impalpha3/impbeta attenuates its cytosolic toxicity in neurons. J. Neurochem., 103, 408-422.

8. Lam, B.D., Anthony, E.C., and Hordijk, P.L. (2013) Cytoplasmic targeting of the protooncogene SET promotes cell spreading and migration. FEBS. Lett., 587, 111-119.

9. Kon, N., Wang, D., and Gu, W. (2019) Loss of SET reveals both the p53-dependent and the p53-independent functions in vivo. Cell Death Dis., 10, 237-247

10. Puffenberger, E.G., Hu-Lince, D., Parod, J.M., Craig, D.W., Dobrin, S.E., Conway, A.R., Donarum, E.A., Strauss, K.A., Dunckley, T., Cardenas, J.F., et al. (2004) Mapping of sudden infant death with dysgenesis of the testes syndrome (SIDDT) by a SNP genome scan and identification of TSPYL loss of function. Proc. Natl. Acad. Sci. U.S.A., 101, 11689-11694.

11. Vinci, G., Brauner, R., Tar, A., Rouba, H., Sheth, J., Sheth, F., Ravel, C., McElreavey, K. and Bashamboo, A. (2009) Mutations in the TSPYL1 gene associated with 46,XY disorder of sex development and male infertility. Fertil. Steril., 92, 1347-1350.

12. Javaher, P., Stuhrmann, M., Wilke, C., Frenzel, E., Manukjan, G., Grosshenig, A., Dechend, F., Schwaab, E., Schmidtke, J. and Schubert, S. (2012) Should TSPYL1 mutation screening be included in routine diagnostics of male idiopathic infertility? Fertil. Steril., 97, 402-406.

13. Hering, R., Frade-Martinez, R., Bajanowski, T., Poets, C.F., Tschentscher, F. and Riess, O. (2006) Genetic investigation of the TSPYL1 gene in sudden infant death syndrome. Genet. Med., 8, 55-58. 
14. Schubert, S., Haas, C., Bartsch, C., Mirshekarnejad, M., Kohrs, S., Roettinger, I., Grosshennig, A., Stuhrmann, M., Scholz, C. and Schmidtke, J. (2015) Variants in TSPYL1 are not associated with sudden infant death syndrome in a cohort of deceased infants from Switzerland. Mol. Cell. Probes, 29, 31-34.

15. Slater, B., Glinton, K., Dai, H., Lay, E., Karaviti, L., Mizerik, E., Murali, C.N., Lalani, S.R., Bacino, C.A. and Rossetti, L.Z. (2020) Sudden infant death with dysgenesis of the testes syndrome in a non-Amish infant: A case report. Am. J. Med. Genet. A., Online ahead of print.

16. Westbury, S.K., Turro, E?, Greene, D., Lentaigne, C., Kelly, A.M., Bariana ,T.K., Simeoni, I., Pillois, X., Attwood, A., Austin, S., et al. (2015) Human phenotype ontology annotation and cluster analysis to unravel genetic defects in 707 cases with unexplained bleeding and platelet disorders. Genome Med., 7, 36-50.

17. Di Michele, M., Peeters, K., Loyen, S., Thys, C., Waelkens, E., Overbergh, L., Hoylaerts, M., Van Geet, C. and Freson, K. (2012) Pituitary Adenylate CyclaseActivating Polypeptide (PACAP) impairs the regulation of apoptosis in megakaryocytes by activating NF-кB: a proteomic study. Mol. Cell. Proteomics, 11, M111.007625.

18. Huang, C.J. Tu, C.T., Hsiao, C.D., Hsieh, F.J. and Tsai, H.J. (2003) Germ-line transmission of a myocardium-specific GFP transgene reveals critical regulatory elements in the cardiac myosin light chain 2 promoter of zebrafish. Dev. Dyn., 228, 30-40.

19. Masai, I., Stemple, D.L., Okamoto, H. and Wilson, S.W. (2000) Midline signals regulate retinal neurogenesis in zebrafish. Neuron, 27, 251-263. 
20. Glasgow, E., Karavanov, A.A. and Dawid, I.B. (1997) Neuronal and neuroendocrine expression of lim3, a LIM class homeobox gene, is altered in mutant zebrafish with axial signaling defects. Dev. Biol., 192, 405-419.

21. Louwette, S., Labarque, V., Wittevrongel, C., Thys, C., Metz, J., Gijsbers, R., Debyser, Z., Arnout, J., Van Geet, C. and Freson, K. (2012) Regulator of G-protein signaling 18 controls megakaryopoiesis and the cilia-mediated vertebrate mechanosensory system. FASEB J., 26, 2125-2136

22. Kay, J.N., Link, B.A. and Baier, H. (2005) Staggered cell-intrinsic timing of ath5 expression underlies the wave of ganglion cell neurogenesis in the zebrafish retina. Development, 132, 2573-2585.

23. Gong, H., Yan, Y., Fang, B., Xue, Y., Yin, P., Li, L., Zhang, G., Sun, X., Chen, Z., Ma, H,. et al. (2014) Knockdown of nucleosome assembly protein 1-like 1 induces mesoderm formation and cardiomyogenesis via notch signaling in murine-induced pluripotent stem cells. Stem Cells, 32, 1759-1773.

24. Sun, S., Li, X., Liu, Z., Zhang, G., Yang, C., Jiang, Q. and Zou, Y. (2020) Expression of nucleosome assembly protein 1 like genes in zebrafish embryos. Gene Expr. Patterns, 35, 119076.

25. Meek, f. (1983) Functional anatomy of the tectum mesencephali of the goldfish. An explorative analysis of the functional implications of the laminar structural organization of the tectum. Brain Res., 287, 247-297.

26. Tao, K.P., Fong, S.W., Lu, Z., Ching, Y.P., Chan, K.W. and Chan, S.Y. (2011) TSPYL2 is important for G1 checkpoint maintenance upon DNA damage. PLoS One, 6, e21602. 
27. Canela, N., Rodriguez-Vilarrupla, A., Estanyol, J.M., Diaz, C, Pujol, M.J., Agell, N. and Bachs, O. (2003) The SET protein regulates G2/M transition by modulating cyclin Bcyclin-dependent kinase 1 activity. J. Biol. Chem., 278, 1158-1164.

28. Canela, N., Rodriguez-Vilarrupla, A., M., Estanyol, J. M., Diaz, C., Pujol, M. J., Agell, N. and Bachs, O. (2003). The SET Protein Regulates G2/M Transition by Modulating Cyclin B-Cyclin-dependent Kinase 1 Activity. J. Biol. Chem., 278, 1158-1164.

29. Kellogg, D. R. , Kikuchi, A., Fuji-Nakata, T., Turck, C. W. and Murray, A. W. (1995). Members of the NAP/SET Family of Proteins Interact Specifically with B-Type cyclins. J. Cell Biol., 130, 661-673.

30. Li, Y., and Lau, Y.F.C. (2008) TSPY and its X-encoded homologue interact with cyclin B but exert contrasting functions on cyclin-dependent kinase 1 activities. Oncogene, 27, 6141-6150.

31. Schafer, K.A. (1998) The cell cycle: a review. Vet. Pathol., 35, 461-478.

32. Scherl, A., Couté, Y., Déon, C., Calle, A., Kindbeiter, K., Sanchez, J.C., Greco, A., Hochstrasser, D. and Diaz, J.J. (2002) Functional proteomic analysis of human nucleolus. Mol. Biol. Cell, 13, 4100-4109.

33. Zhivotovsky, B.and Orrenius, S. (2010) Cell cycle and cell death in disease: past, present and future. J. Intern. Med., 268, 395-409.

34. Karaca, E., Posey, J.E., Bostwick, B., Liu, P., Gezdirici, A., Yesil, G., Coban Akdemir, Z., Bayram, Y., Harms, F.L., Meinecke, P. et al. (2019) Biallelic and De Novo Variants in DONSON Reveal a Clinical Spectrum of Cell Cycle-opathies with Microcephaly, Dwarfism and Skeletal Abnormalities. Am. J. Med. Genet. A., 179, 2056-2066. 


\section{Legends to figures}

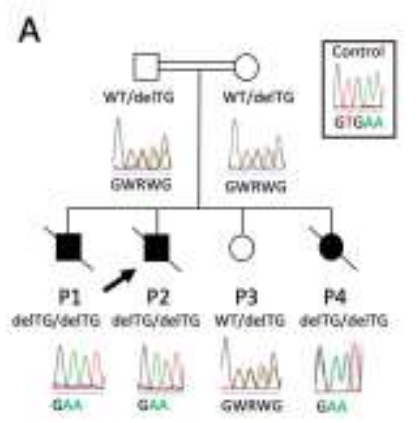

B

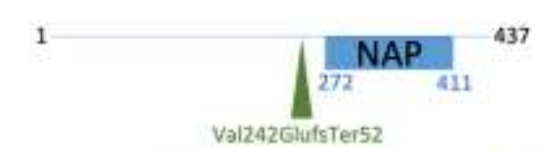

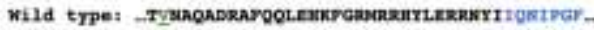

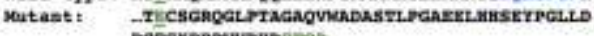

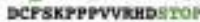

C

D

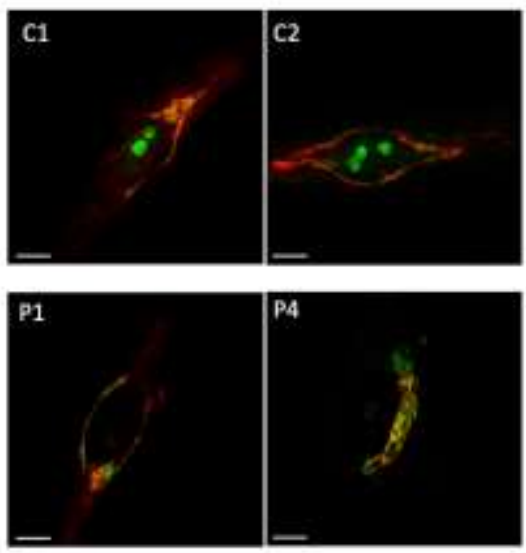

Figure 1. Characterization of the TSPYL1 variant at DNA and protein level. A. Pedigree with three affected (P1, P2 and P4) children who died of cardiac arrest before the first year of life and one healthy sibling (P3). The patients are homozygous for c.725_726delTG in TSPYL1 while parents and P4 are heterozygous. B. The frameshift variant is predicted to result in a truncated protein p.Val242GluTer52 with an early STOP codon and a frameshift that disrupts the NAP 
domain of TSPYL1. C. Immunoblot analysis extracts from healthy control (C) fibroblasts show TSPYL1 expression in the nuclear fraction with predicted molecular weight of 49kDa. Fibroblast extracts from the patients express a shorter protein that mainly remains in the cytoplasm faction. The HDAC1 antibody was used to show enrichment of nuclear proteins in the nuclear fraction. $\mathrm{M}$, Molecular weight (kDa). D. Immunofluorescent staining of TSPYL1 (green) and mannosidase (red) shows retention of the protein in the Golgi of patients' fibroblasts while control fibroblasts express TSPYL1 in the nucleoli. Images are representative for all analysed patient fibroblasts.

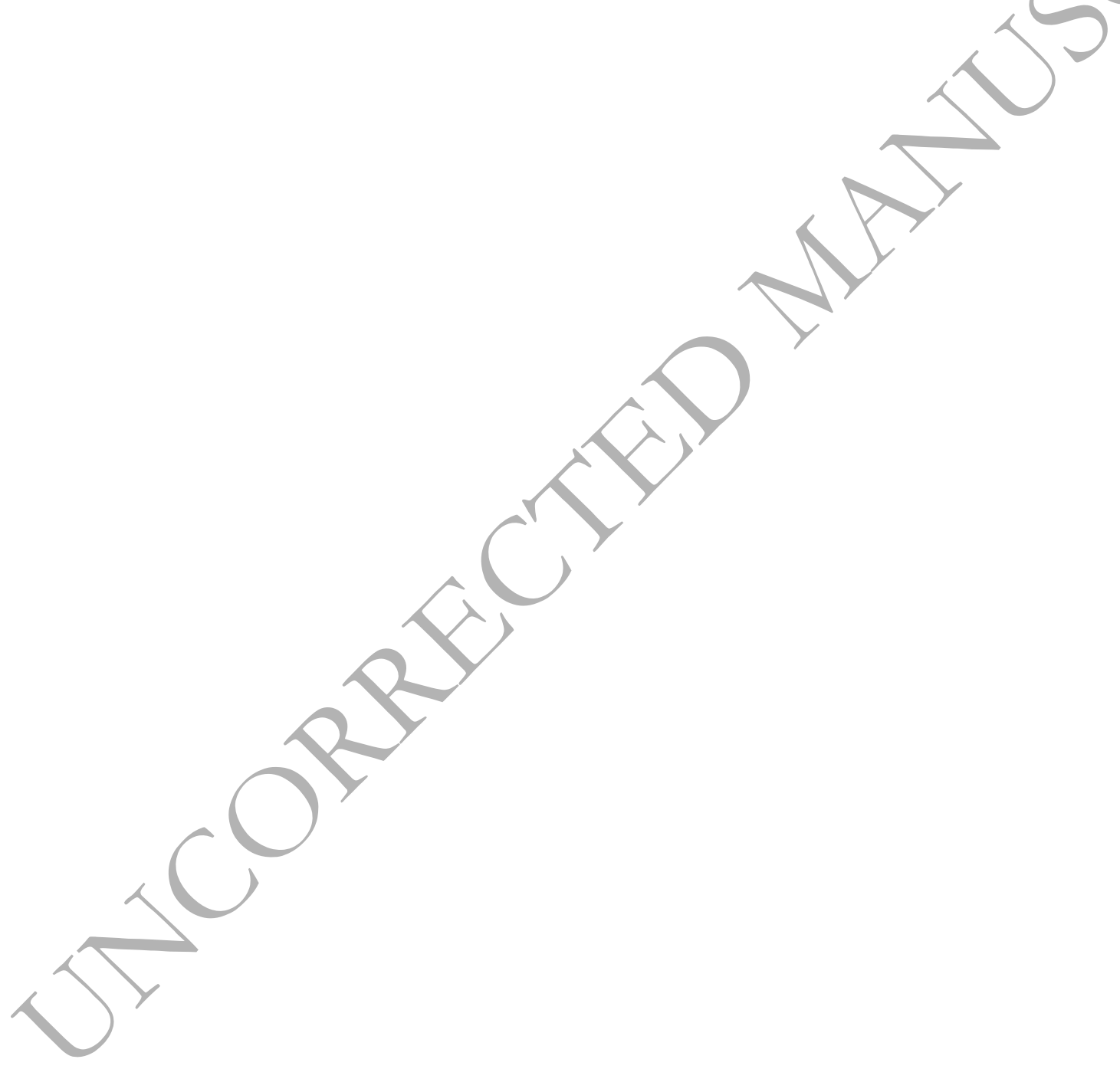


A

48 differentially expressed spots $(p<0.05, P / C 1.2)$

П 25 Upregulated

423 Downregulated

MS/MS identification

24 Upregulated

20 Downregulated

35 unique identified proteins

of which 15 proven nuclear expression

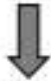

Pathway analysis

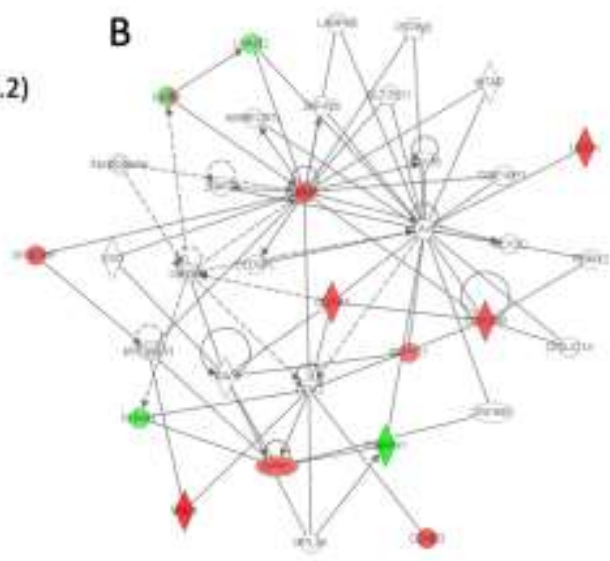

C
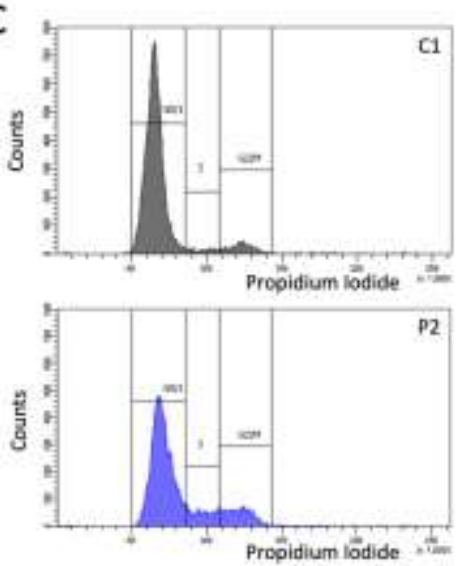

D

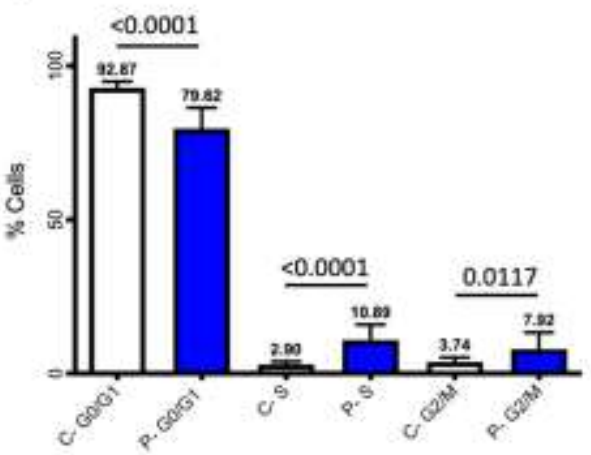

E

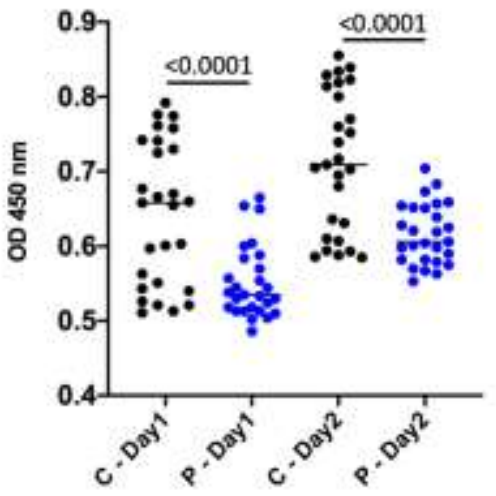

Figure 2. TSPYL1 and cell cycling. A. Experimental overview of the 2-DIGE experiment used to analyze nuclear extracts from fibroblasts of five controls and three patients and identification of differentially expressed proteins (full list in Table 1). B. The 15 proteins differentially 
expressed between patients and controls and annotated as nuclear were used as input for analysis of regulatory networks using IPA (ingenuity Pathway Analysis). The "cell cycle" pathway showed is the highest-ranked. Nodes represent proteins: colored features depict proteins identified in the present study (green: downregulated/red: upregulated, details in Table 1) whereas un-shaded features depict additional members of this network that were not detected by DIGE/MS. Node shapes indicate function: enzymes (diamond), transcription regulators (oval), nuclear receptors (rectangle), cytokines (square), transporter (trapezoid) and "other" (circles). C. Flow cytometric analysis of the cell cycle using propidium iodide staining for fibroblasts of three controls and three patients. Representative profiles with G0/1, S and G2/M phases are shown for control C1 and patient P2. D. Quantifications of triplicate flow cytometric analysis of the cell cycle for fibroblasts of three unrelated controls and three patients. Each triplicate experiment was measured in duplicate (total of six values for each individual fibroblast line). The $\%$ of cells in G0/1, S and G2/M phases are shown as mean for three controls and three patients with standard deviations. Statistical analysis was performed by one-way ANOVA with Bonferroni's multiple comparisons test. E. Cell proliferation assay for fibroblasts cultured in 96 wells (20.000 cells/well) after 1 and 2 days of growth was assessed by a WST-1-based cell viability assay. Data are expressed as mean and standard deviation $(\mathrm{n}=9$ for each of the three controls and three patients in a triplicate experiment). Statistical analysis was performed by one-way ANOVA with Tukey's multiple comparisons test. 

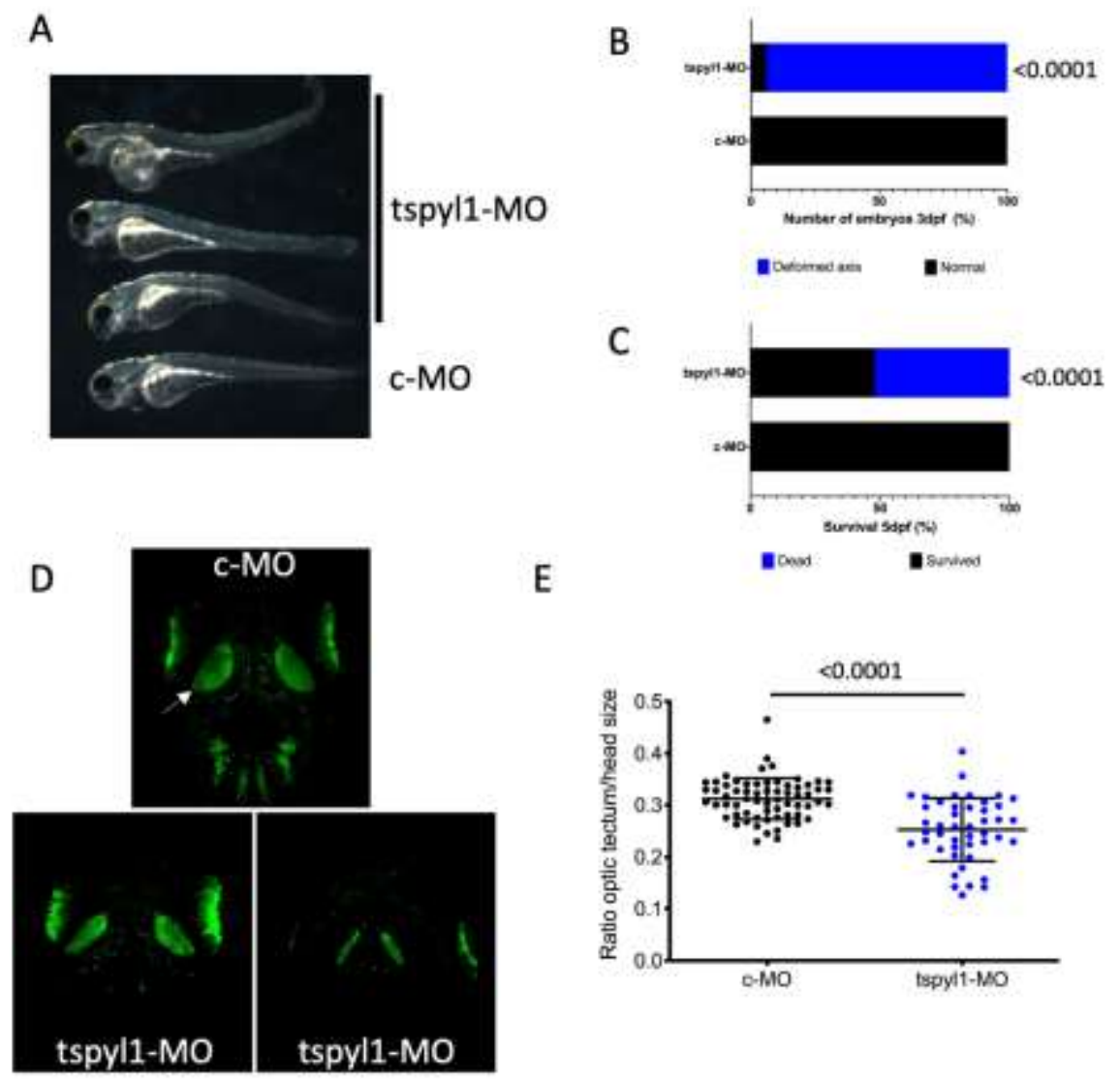

E

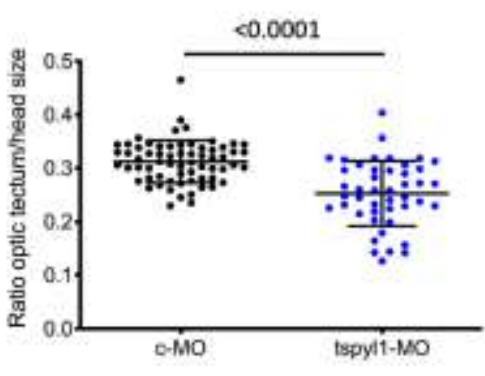

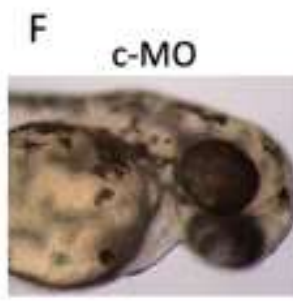

$\mathrm{H}$

c-MO

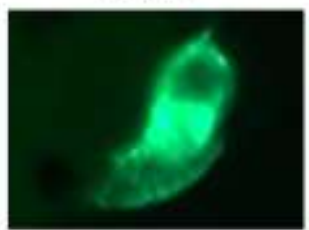

tspyl1-MO

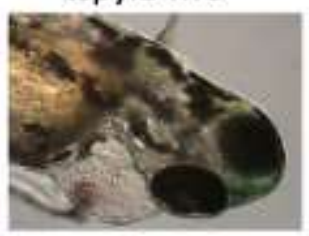

tspyl1-MO

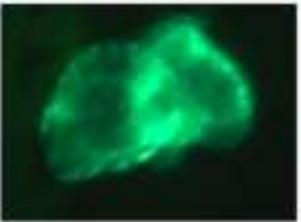

G

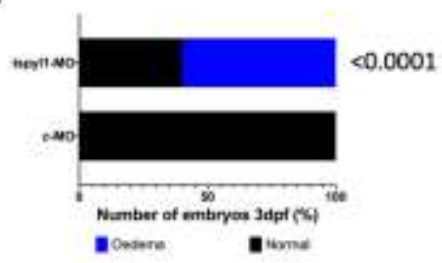

1

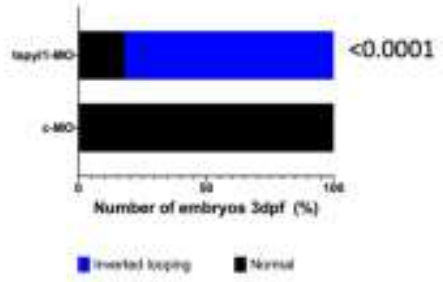

Figure 3. Tspyl1 depletion in Zebrafish embryos. A. Representative images of AB embryos injected with tspyl1-MO at $3 \mathrm{dpf}$ with deformed axes in contrast to c-MO injected embryos. B. Life screening of $87 \mathrm{AB}$ embryos (from duplicated experiment) showed $94 \%$ of deformed embryos after tspyl1 depletion while deformed axes could not be detected in c-MO injected 
embryos. Statistical analysis was performed by Fisher's exact test. C. Survival analysis of 261 tspyl1-MO and $273 \mathrm{c}-\mathrm{MO}$ injected $\mathrm{AB}$ embryos (from 4 experiments) at 5 dpf showed $52 \%$ dead fish for tspyl1 depleted embryos and $11 \%$ for control embryos. Statistical analysis was performed by Fisher's exact test. D. Representative images of the optic tectum (arrows) in transgenic Tg (ath5:eGFP) embryos after injection with c-MO or tspyl1-MO at 5dpf. E. Quantification of the optic tectum diameter normalized by the head size for c-MO ( $\mathrm{n}=64,4$ injections) or tspyl1-MO $(\mathrm{n}=44,3$ injections) injected embryos at 5dpf. Dots represent measurements in single embryos and the data are presented as mean and standard deviation. Statistical analysis was performed by Mann Whitney test. F. Representative images of transgenic $\operatorname{Tg}($ cmcl2:eGFP) embryos that present with heart edema at 3dpf after tspyl1 depletion. G. Quantification of 25 tspyl1-MO and $24 \mathrm{c}-\mathrm{MO}$ injected $\operatorname{Tg}(\mathrm{cmcl} 2: e G F P)$ embryos (from 1 experiment) showed edema formation near the heart in $60 \%$ of tspyl1-MO injected embryos at 3 dpf. Statistical analysis was performed by Mann Whitney test. H. Representative images of transgenic $\operatorname{Tg}(\mathrm{cmcl2}$ :eGFP) embryos showed a dilated heart with inverted looping at 3dpf after tspyl1 depletion. I. Quantification of 80 tspyl1$\mathrm{MO}$ and 76 c-MO injected $\operatorname{Tg}(c m c l 2: e G F P)$ embryos (from 3 experiments) showed defective heart looping in $682 \%$ of tspyl $-\mathrm{MO}$ injected embryos at 3 dpf. Statistical analysis was performed by Mann Whitney test.

Table 1. Proteins from nuclear extracts differentially expressed (red up- and green downregulation) in patients $(\mathrm{P})$ compared to controls $(\mathrm{C})(\mathrm{p} \leq 0.05, \mathrm{P} / \mathrm{C}$ fold change $\geq 1.2)$

\begin{tabular}{llllllll}
\hline Sp & T- & P/C & Identification & SwissP & M & Decoy & Matchi \\
ot & test & Rat & & rot & W & MASC & ng \\
$\mathbf{N}^{\circ}$ & & io & & code & (kD & OT & peptid \\
& & & & a) & Score & es \\
\hline
\end{tabular}




\section{Upregulated in fibroblasts from patients}

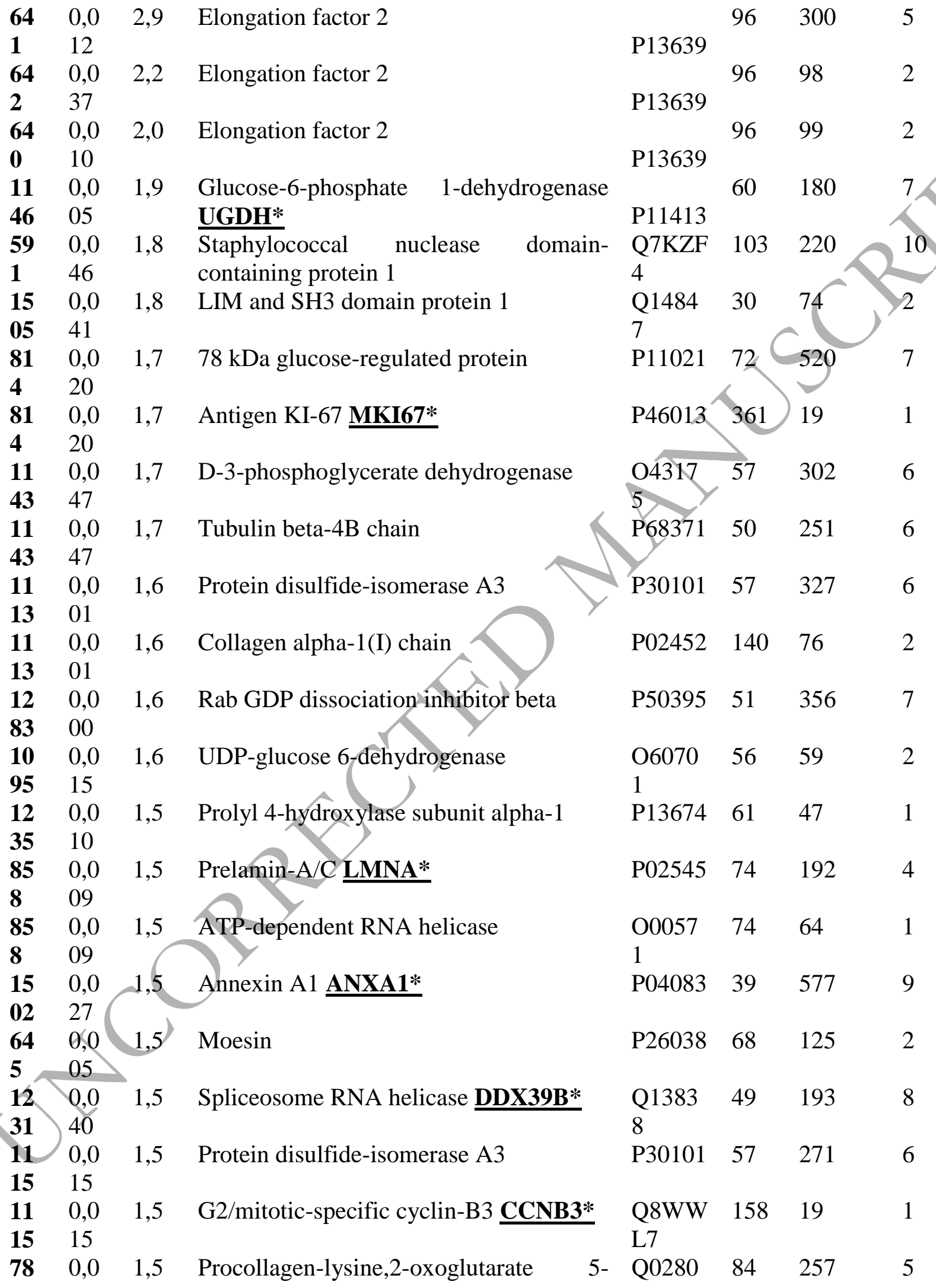


$\begin{array}{llll}3 & 42 & \text { dioxygenase } 1 & 9\end{array}$

$12 \begin{array}{lllllllll} & 0,0 & 1,4 & \text { Prolyl 4-hydroxylase } & \text { subunit alpha-1 } & \text { P13674 } & 61 & 231 & 4\end{array}$

$2633 \quad$ P4HA2*

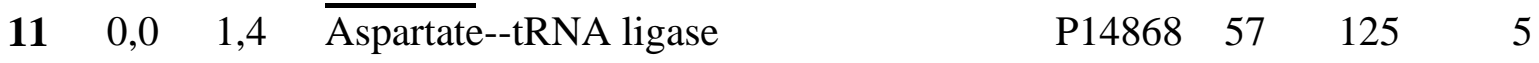

$\mathbf{7 1} 12$

$92 \quad 0,0 \quad 1,4 \quad$ Heterogeneous nuclear ribonucleoprotein

$7 \quad 27 \quad$ Q

$10 \quad 0,0 \quad 1,3 \quad$ Prolyl 4-hydroxylase subunit alpha-2

$2143 \quad 0,0 \quad 1,3 \quad$ Heterogeneous nuclear

$\begin{array}{lllllll}\mathbf{1 7} & 0,0 & 1,3 & \text { Heterogeneous nuclear ribonucleoprotein } & \text { P31942 } & 37 & 247\end{array}$

$36 \quad 22$ H3

$17 \quad 0,0 \quad 1,3 \quad$ Nucleophosmin $\underline{\text { NPM* }}$

3622

$12 \quad 0.0 \quad 1.2 \quad$ Septin 11

1954

O6050 $70 \quad 435 \quad 7$

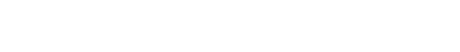

Downregulated in fibroblasts from patients

$12 \quad 0,0 \quad-1,3 \quad$ Actin-related protein 3

4623

$94 \quad 0,0 \quad-1,4 \quad$ Lamin-B2 LMNB2*

$9 \quad 37$

$13 \quad 0,0 \quad-1,4 \quad$ Tubulin alpha-1C chain

$97 \quad 49$

$13 \quad 0,0 \quad-1,4 \quad$ Tubulin alpha-8 chain

9749

$63 \quad 0,0 \quad-1,5 \quad$ Alpha-actinin-1

O1546 $61 \quad 165$

$\mathrm{P} 06748 \quad 33 \quad 49 \quad 3$

$5 \quad 28$

$11 \quad 0,0-1,5 \quad$ Vimentin VIME*

8938

$13 \quad 0,0 \quad-1,7$ Actin, cytoplasmic 1 ACTB*

9441

$15 \quad 0,0 \quad-1,7 \quad$ Vimentin

P06748 $33>^{49}$

2709

$15 \quad 0,0 \quad-1,7 \quad$ Tropomyosin alpha-1

2709

$15 \quad 0,0 \quad-1,7$ Tropomyosin beta chain

$27 \quad 09$

$150,0 \quad-1,7$ Tropomyosin alpha-3 chain

$27 \quad 09$

$15 \quad 0,0-1,8 \quad$ Tropomyosin alpha-4 chain

$78 \quad 26$

$150,0 \quad-1,8 \quad$ Vimentin

7826

$17 \quad 0,0 \quad-1,8$ Tropomyosin alpha-1 chain

3516

$17 \quad 0,0 \quad-1,8$ Tropomyosin alpha-3 chain

3516

\begin{tabular}{|c|c|c|c|}
\hline \multicolumn{4}{|l|}{ P61158 } \\
\hline Q0325 & 68 & 62 & \\
\hline $\begin{array}{l}\text { Q9BQ } \\
\text { E3 }\end{array}$ & 51 & 62 & \\
\hline $\begin{array}{l}\text { Q9NY } \\
65\end{array}$ & 51 & 37 & \\
\hline P12814 & 103 & 119 & \\
\hline P08670 & 54 & 243 & \\
\hline P60709 & 42 & 136 & \\
\hline P08670 & 54 & 103 & \\
\hline P09493 & 33 & 100 & \\
\hline P07951 & 33 & 78 & \\
\hline P06753 & 33 & 76 & \\
\hline P67936 & 29 & 113 & \\
\hline P08670 & 54 & 70 & \\
\hline P09493 & 33 & 68 & \\
\hline P06753 & 33 & 44 & \\
\hline
\end{tabular}


$17 \quad 0,0 \quad-1,8 \quad$ Tropomyosin beta chain

3516

$17 \quad 0,0 \quad-1,8 \quad$ Vimentin

P07951 $33 \quad 43 \quad 2$

3516

$110,0 \quad-1,9 \quad$ Vimentin

$\begin{array}{llll}\mathrm{P} 08670 & 54 & 28 & 1\end{array}$

$47 \quad 42$

$11 \quad 0,0 \quad-1,9$ Nuclear mitotic apparatus protein 1

$47 \quad 42$ NUMA1*

$11 \quad 0,0 \quad-2,0 \quad \frac{\text { Vimentin }}{23}$

$\begin{array}{llll}\mathrm{P} 08670 & 54 & 693 & 9\end{array}$

2312

$11 \quad 0,0 \quad-2,0 \quad$ Vimentin

1402

$13 \quad 0,0 \quad-2,0 \quad$ Actin, cytoplasmic 1

Q1498 $240 \quad 30$

5249

$13 \quad 0,0 \quad-2,0 \quad$ Actin, alpha cardiac muscle 1

5249

$13 \quad 0,0 \quad-2,0 \quad$ Beta-actin-like protein 2

5249

$11 \quad 0,0 \quad-2,1 \quad$ Vimentin

0

1923

$110,0 \quad-2,1$ Acyl-CoA dehydrogenase family

1923 member 10

$13 \quad 0,0 \quad-2,1 \quad$ Actin, cytoplasmic 1

P08670 $54 \quad 828$

P08670 $54 \quad 776$

6014

$13 \quad 0,0 \quad-2,1 \quad$ Actin, alpha cardiac muscle 1

6014

$13 \quad 0,0 \quad-2,1 \quad$ Beta-actin-like protein 2

6014

$10 \quad 0,0 \quad-2,1 \quad$ Vimentin

9804

$10 \quad 0,0 \quad-2,1 \quad$ Tetratricopeptide repeat protein 17

9804

$11 \quad 0,0 \quad-2,1 \quad$ Vimentin

P60709 42400

P68032 $42 \quad 267 \quad 4$

Q562R $42 \quad 183 \quad 2$

1

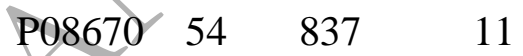

0815

$17 \quad 0,0 \quad-2,3 \quad$ Actin, cytoplasmic 1

$34 \quad 07$

$17 \quad 0,0 \quad-2,3 \quad$ Beta-actin-like protein 2

$34 \quad 07$

$120,0-2,5$ Polyadenylate-binding protein

$14 \quad 12 \quad$ PABP2*

$130,0 \quad-2,5$ Actin, cytoplasmic 1

Q6JQN

$120 \quad 35 \quad 2$

1

P60709 $42 \quad 433 \quad 10$

$\begin{array}{llll}\text { P68032 } & 42 & 246 & 4\end{array}$

Q562R $42 \quad 172 \quad 2$

1

$\begin{array}{llll}\mathrm{P} 08670 & 54 & 392 & 10\end{array}$

$\begin{array}{llll}\text { Q96AE } & 130 & 27 & 1\end{array}$

7

$\begin{array}{llll}\mathrm{P} 08670 & 54 & 1101 \quad 15\end{array}$

$\begin{array}{llll}\text { P60709 } & 42 \quad 73 \quad 3\end{array}$

Q562R $42 \quad 56 \quad 2$

1

$54 \quad 03$

$13 \quad 0,0 \quad-2,5 \quad$ Beta-actin-like protein 2

5403

*Nuclear proteins used as input list for the enrichment pathway analysis 


\section{Abbreviations}

NAP nucleosome assembly protein

TSPYL1 TSPY-like 1

TTSN TSPY/TSPYL/SET/NAP1

SIDDT sudden infant death with dysgenesis of the testes

MO

Ath5

$\mathrm{Tg}$

dpf

FDR morpholino

atonal-homologue 5

transgenic

days post fertilization

false discovery rate

IPA

ingenuity Pathway Analysis

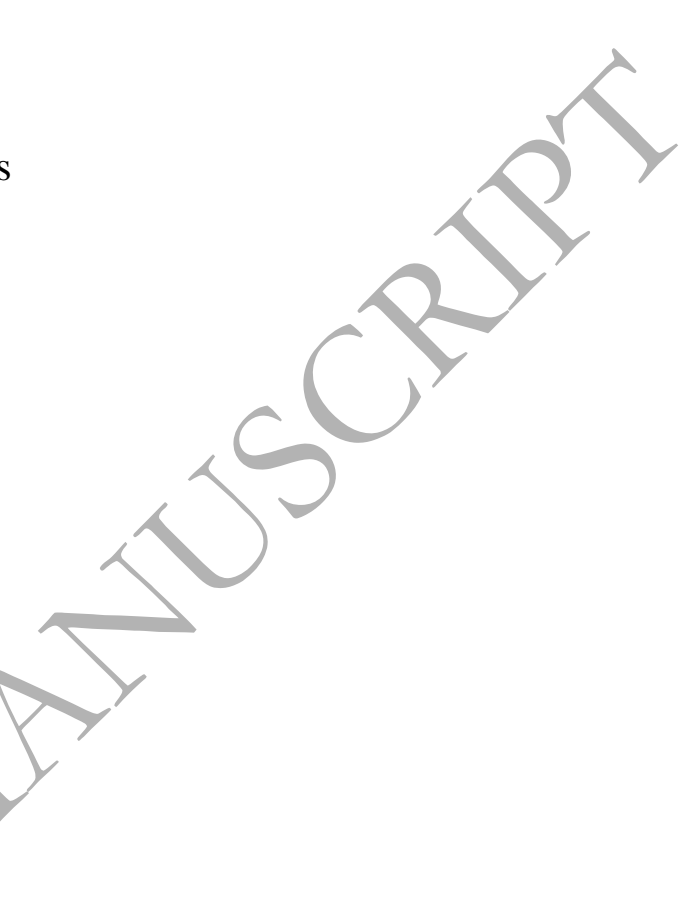

